\title{
Solution to the equations of the moment expansions
}

Research Article

\author{
Paolo Amore ${ }^{1 *}$, Francisco M. Fernández ${ }^{2 \dagger}$ \\ 1 Facultad de Ciencias, CUICBAS, Universidad de Colima, \\ Bernal Díaz del Castillo 340, Colima, Colima, Mexico \\ 2 INIFTA (UNLP, CCT La Plata-CONICET), División Química Teórica, \\ Blvd. 113 S/N, Sucursal 4, Casilla de Correo 16, 1900 La Plata, Argentina
}

Received 30 August 2012; accepted 20 October 2012

\begin{abstract}
:
We develop a formula for matching a Taylor series about the origin and an asymptotic exponential expansion for large values of the coordinate. We test it on the expansion of the generating functions for the moments and connected moments of the Hamiltonian operator. In the former case the formula produces the energies and overlaps for the Rayleigh-Ritz method in the Krylov space. We choose the harmonic oscillator and a strongly anharmonic oscillator as illustrative examples for numerical test. Our results reveal some features of the connected-moments expansion that were overlooked in earlier studies and applications of the approach.
\end{abstract}

PACS (2008): 03.65.Ge

Keywords: $\quad$ connected moments expansion $\bullet$ full solution $•$ convergence $\cdot$ Rayleigh-Ritz method

(c) Versita sp. z o.o.

\section{Introduction}

Some time ago Horn and Weinstein [1] and Horn et al. [2] proposed the calculation of the ground-state energy of quantum-mechanical systems by means of the Taylor expansion of the generating function for the cumulants or connected moments. The main problem of this approach is the extrapolation of the $t$-expansion for $t \rightarrow \infty$. Those authors proposed approximate expressions based on Padé approximants that did not produce encouraging results. For that reason Cioslowski [3] suggested the extrapolation by means of a series of exponential functions. This

*E-mail: paolo.amore@gmail.com

†E-mail: fernande@quimica.unlp.edu.ar (Corresponding author) and other approaches were discussed and compared by Stubbins [4]. Cioslowski's approach leads to a nonlinear system of equations for the parameters in the exponential expansion that he solved by means of a systematic algorithm that avoids the explicit calculation of the unnecessary variables. The resulting approach has since been known as the connected-moments expansion or CMX. Later, Knowles [5] developed an elegant expression for the CMX approximants to the energy of the ground state in terms of matrices built from the connected moments. Since then, the $C M X$ has been applied to a wide variety of problems and has been extensively discussed and generalized. A complete list of references is given elsewhere [6]; here we restrict ourselves to those papers that are relevant to the present discussion.

In a recent paper Amore et al. [6] analyzed the CMX by means of simple quantum-mechanical models and conjec- 
tured that the parameters in the exponential expansion proposed by Cioslowski [3] may give a clue on the success of the approach. However, the seminal papers on the CMX $[3,5]$ as well as all the later applications of the method (see, for example, the references in [6]) were focused on the calculation of the energy avoiding the explicit calculation of the parameters of the exponential expansion. The main purpose of this paper is to provide the full solution to the nonlinear equations that appear in the problem of matching a Taylor series about the origin and an asymptotic exponential expansion at infinity. In particular, we specialize in the CMX equations because the exponential parameters proved useful to predict the success of the approach [6]. In order to test the main equations to any desired order of approximation we select simple quantum-mechanical models that allow the calculation of a sufficiently great number of connected moments. In Section 2 we develop the main equations for the general problem of matching the two asymptotic series. In Section 3 we apply these equations to the generating functions for the moments and connected moments. In Section 4 we conduct a numerical test on simple quantum-mechanical models. Finally, in Section 5 we discuss the results, draw conclusions and propose further applications of the main equations.

\section{Matching the expansions}

Suppose that a function $F(t)$ can be expanded in a formal power series for small $t$

$$
F(t)=\sum_{j=0}^{\infty} \frac{(-t)^{j}}{j !} F_{j}
$$

and in an exponential expansion for large $t$

$$
F(t)=\sum_{j=0}^{\infty} d_{j} e^{-t e_{j}}
$$

where $\operatorname{Re}\left(e_{j}\right)>0$. We can match both expansions at $t=0$ provided that the series in the right-hand side of the equations

$$
F_{k}=\sum_{j=0}^{\infty} d_{j} e_{j}^{k}, k=0,1, \ldots
$$

converge.

We are interested in the case that we do not know the parameters $d_{j}$ and $e_{j}$ of the exponential expansion. Therefore, we try an ansatz of the form

$$
F^{(N)}(t)=\sum_{j=0}^{N-1} d_{j} e^{-t e_{j}}
$$

and match its Taylor expansion about $t=0$ with the actual power series (1). In this way we obtain the following system of $2 \mathrm{~N}$ nonlinear equations with the $2 \mathrm{~N}$ unknowns $d_{j}$ and $e_{j}$ :

$$
F_{k}=\sum_{n=0}^{N-1} d_{n} e_{n}^{k}, k=0,1, \ldots, 2 N-1
$$

Strictly speaking, we should write $d_{n, N}$ and $e_{n, N}$ because these parameters depend on the number of terms in the ansatz. However, we just write $d_{n}$ and $e_{n}$ to keep the notation simpler.

In order to solve equations (5) we consider the auxiliary system of $N$ linear equations with $N$ unknowns $c_{i}$

$$
\sum_{i=0}^{N-1}\left(F_{i+j+1}-W F_{i+j}\right) c_{i}=0, j=0,1, \ldots, N-1 .
$$

There are nontrivial solutions only if its determinant vanishes

$$
\left|F_{i+j+1}-W F_{i+j}\right|_{i, j=0}^{N-1}=0
$$

and $W$ is one of the $N$ roots $W_{0}, W_{1}, \ldots, W_{N-1}$ of the characteristic polynomial

$$
p(W)=\sum_{j=0}^{N} p_{j} W^{j}=0
$$

given by Eq. (7).

If we define

$$
\gamma_{j}=\sum_{i=0}^{N-1} F_{i+j} c_{i}, j=0,1, \ldots, N-1
$$

then equations (6) reduce to $\gamma_{j+1}=W \gamma_{j}$, $j=0,1, \ldots, N-1$ and $\gamma_{j}=W^{j} \gamma_{0}$, so that

$$
\sum_{j=0}^{N} \gamma_{j} p_{j}=\sum_{i=0}^{N-1} c_{i} \sum_{j=0}^{N} F_{i+j} p_{j}=\gamma_{0} \sum_{j=0}^{N} p_{j} W^{j}=0 .
$$

Taking into account Eq. (5) it is clear that

$$
\sum_{j=0}^{N} F_{i+j} p_{j}=\sum_{n=0}^{N-1} d_{n} e_{n}^{i} \sum_{j=0}^{N} p_{j} e_{n}^{j}
$$

Therefore, if we choose the exponential parameters to be the roots of the pseudo-secular determinant (7): $e_{n}=W_{n}$, 
$n=0,1, \ldots, N-1$ we then conclude that the coefficients $p_{j}$ of the characteristic polynomial (8) are given by

$$
\sum_{j=0}^{N} F_{i+j} p_{j}=0, i=0,1, \ldots, N-1
$$

If, without loss of generality, we choose $p_{0}=1$, then the remaining polynomial coefficients $p_{1}, p_{2}, \ldots, p_{N}$ are the solutions to the nonhomogeneous linear system of equations

$$
\begin{aligned}
\left(\begin{array}{llll}
F_{1} & F_{2} & \cdots & F_{N} \\
F_{2} & F_{3} & \cdots & F_{N+1} \\
\vdots & \vdots & \ddots & \vdots \\
F_{N} & F_{N+1} & \cdots & F_{2 N-1}
\end{array}\right) & \left(\begin{array}{l}
p_{1} \\
p_{2} \\
\vdots \\
p_{N}
\end{array}\right)= \\
= & \left(\begin{array}{l}
F_{0} \\
F_{1} \\
\vdots \\
F_{N-1}
\end{array}\right)
\end{aligned}
$$

provided that the square matrix $\mathrm{F}=\left(F_{i+j+1}\right)_{i, j=0}^{N-1}$ is nonsingular. Otherwise the truncated Taylor (1) and exponential (2) expansions cannot be successfully matched at origin.

Once we have the roots of $p(W)=0$ the nonlinear equations (5) become linear equations for the remaining unknowns $d_{n}$. There are $2 N$ such equations but we only need $N$ of them; for concreteness we arbitrarily choose the first $N$ ones. The occurrence of multiple roots $e_{n}=W_{n}$ reduces the order $N$ of the ansatz $F^{(N)}(t)$.

The starting point of present proof Eq. (6) was motivated by an earlier paper where Fernández [7] proved the equivalence between the Rayleigh-Ritz variation method in the Krylov space and the connected-moments polynomial approach [8].

\section{Generating functions for the mo- ments and connected moments}

The expansion of the generating function

$$
Z(t)=\left\langle\phi\left|e^{-t \hat{H}}\right| \phi\right\rangle
$$

in a Taylor series about the origin

$$
Z(t)=\sum_{j=0}^{\infty} \frac{(-t)^{j}}{j !} \mu_{j}
$$

yields the moments $\mu_{j}=\left\langle\phi\left|\hat{H}^{j}\right| \phi\right\rangle$ of the Hamiltonian operator $\hat{H}$ with respect to a trial or reference state $|\phi\rangle$. If the spectrum of $\hat{H}$ is discrete

$$
\hat{H}\left|\psi_{j}\right\rangle=E_{j}\left|\psi_{j}\right\rangle, j=0,1, \ldots
$$

and its eigenfunctions form a complete set then

$$
Z(t)=\sum_{j=0}^{\infty}\left|\left\langle\phi \mid \psi_{j}\right\rangle\right|^{2} e^{-t E_{j}}
$$

provided that $\left\langle\psi_{i} \mid \psi_{j}\right\rangle=\delta_{i j}$. Therefore, we can apply the method developed in the preceding section with $F_{j}=\mu_{j}$. For concreteness we assume that $E_{0} \leq E_{1} \leq E_{2} \leq \ldots$.

In this case equations (6) and (7) are the secular equations and secular determinant, respectively, for the RayleighRitz method in the Krylov space [7] (and references therein). Therefore, the roots $W_{j}$ are real and for each of them we have an approximate solution

$$
\begin{aligned}
\left|\varphi_{j}\right\rangle & =\sum_{i=0}^{N-1} c_{i j}\left|\phi_{i}\right\rangle \\
j & =0,1, \ldots, N-1,\left|\phi_{i}\right\rangle=\hat{H}^{i}|\phi\rangle
\end{aligned}
$$

Without loss of generality we assume that $\left\langle\varphi_{i} \mid \varphi_{j}\right\rangle=\delta_{i j}$. Besides, we know that the approximate variational eigenvalues are upper bounds to the exact ones: $W_{j}^{(N)}>$ $W_{j}^{(N+1)}>E_{j}$.

The projection operator

$$
\hat{P}_{N}=\sum_{j=0}^{N-1}\left|\varphi_{j}\right\rangle\left\langle\varphi_{j}\right|
$$

satisfies

$$
\hat{P}_{N}\left|\phi_{i}\right\rangle=\left|\phi_{i}\right\rangle, \quad i=0,1, \ldots, N-1 .
$$

For the projected Hamiltonian

$$
\hat{H}_{N}=\hat{P}_{N} \hat{H} \hat{P}_{N}
$$

we have

$$
\hat{H}_{N}^{j}|\phi\rangle=\hat{P}_{N} \hat{H}^{j}|\phi\rangle, j=0,1, \ldots, N .
$$

Therefore

$$
\left\langle\phi\left|\hat{H}_{N}^{j}\right| \phi\right\rangle=\left\langle\phi\left|\hat{H}^{j}\right| \phi\right\rangle, j=0,1, \ldots, 2 N-1 .
$$


The approximate generating function

$$
Z^{(N)}(t)=\left\langle\phi\left|e^{-t \hat{H}_{N}}\right| \phi\right\rangle
$$

exhibits a finite exponential expansion

$$
Z^{(N)}(t)=\sum_{j=0}^{N-1}\left|\left\langle\phi \mid \varphi_{j}\right\rangle\right|^{2} e^{-t W_{j}}
$$

and its Taylor series about $t=0$ yields the first $2 N-1$ exact moments

$$
Z^{(N)}(t)=\sum_{j=0}^{2 N-1} \frac{(-t)^{j}}{j !} \mu_{j}+\ldots
$$

Therefore, if we apply the method of the preceding section the parameters $d_{j}$ and $e_{j}$ of the approximate exponential expansion (4) should be $d_{j}=\left|\left\langle\phi \mid \varphi_{j}\right\rangle\right|^{2}$ and $e_{j}=W_{j}$ if there is no degeneracy. If $W_{j}$ is $m$-fold degenerate then the coefficient $d_{j}$ will be the sum of the corresponding $m$ overlaps $\left|\left\langle\phi \mid \varphi_{j}\right\rangle\right|^{2}$. We think that it is surprising that merely matching an exponential-series ansatz and a Taylor series may lead to the results of the Rayleigh-Ritz method.

The function

$$
E(t)=-\frac{Z^{\prime}(t)}{Z(t)}
$$

is monotonically decreasing [1] and its Taylor expansion about $t=0$ yields the connected moments $l_{j}$ :

$$
E(t)=\sum_{j=0}^{\infty} \frac{(-t)^{j}}{j !} l_{j+1}
$$

that one easily obtains in terms of the moments $\mu_{j}$ by means of the recurrence relation [1]

$$
\begin{aligned}
\iota_{1} & =\mu_{1} \\
l_{j+1} & =\mu_{j+1}-\sum_{i=0}^{j-1}\left(\begin{array}{l}
j \\
i
\end{array}\right) I_{i+1} \mu_{j-i}, j=1,2, \ldots .
\end{aligned}
$$

In order to extrapolate $E(t)$ to $t \rightarrow \infty$ and obtain an approximation to the ground-state energy $E_{0}$ Cioslowski [3] proposed the exponential-series ansatz

$$
E^{(N)}(t)=A_{0}+\sum_{j=1}^{N} A_{j} e^{-b_{j} t}
$$

where the unknown parameters $b_{j}$ are supposed to be real and positive. Matching this expression with the $t$-expansion (28) leads to the set of equations

$$
\begin{aligned}
I_{1} & =\sum_{n=0}^{N} A_{n}, \\
I_{k+1} & =\sum_{n=1}^{N} A_{n} b_{n}^{k}, k=1,2, \ldots, 2 N .
\end{aligned}
$$

Arguing as in the preceding section we conclude that the exponential parameters $b_{j}, j=1,2, \ldots, N$ are the roots of the pseudo-secular determinant

$$
\left|I_{i+j+1}-b I_{i+j}\right|_{i, j=1}^{N}=0 \text {. }
$$

Once we have the exponential parameters we solve $N$ of the remaining linear equations (31) for the coefficients $A_{j}$ and then we obtain $A_{0}$ from the first equation:

$$
A_{0}=I_{1}-\sum_{n=1}^{N} A_{n} .
$$

In order to test the consistency of the main CMX assumption we can try the alternative ansatz

$$
U^{(N)}(t)=\sum_{j=0}^{N} A_{j} e^{-b_{j} t}
$$

and verify that there is a stable root $b_{0}$ that approaches zero as $N$ increases. The corresponding pseudo-secular determinant is slightly different from the previous one:

$$
\left|l_{i+j+2}-b l_{i+j+1}\right|_{i, j=0}^{N}=0 .
$$

It is not unlikely that we find difficulties in matching the Taylor and exponential series for the connected moments because the denominator of $E(t)$ exhibits zeros in the complex $t$-plane $Z(t)=0$. Amore et al. [6] have already discussed this point by means of simple examples and here we will show that present mathematical formulas are of considerable help for that purpose.

In the standard implementation of the CMX one does not calculate the parameters $b_{j}$ explicitly [3]. For example, Knowles [5] derived the following explicit expression for the approximant of order $M$ to the coefficient $A_{0}$ :

$$
\begin{aligned}
A_{0, M}= & I_{1}-\left(\begin{array}{llll}
I_{2} & I_{3} & \cdots & I_{M+1}
\end{array}\right) \\
& \left(\begin{array}{llll}
I_{3} & I_{4} & \cdots & I_{M+2} \\
I_{4} & I_{5} & \cdots & I_{M+3} \\
\vdots & \vdots & \ddots & \vdots \\
I_{M+2} & I_{M+3} & \cdots & I_{2 M+1}
\end{array}\right)^{-1}\left(\begin{array}{l}
I_{2} \\
I_{3} \\
\vdots \\
I_{M+1}
\end{array}\right)
\end{aligned}
$$


where

$$
\lim _{M \rightarrow \infty} A_{0, M}=A_{0}=E_{0}
$$

provided that the method converges. If we compare equations (5) and (31) we realize that $F_{j}=l_{j+2}$ and the matrix $F$ in Eq. (13) is exactly the matrix inverted in Eq. (36). Therefore, the approximant $A_{0, M}$ becomes singular when it is not possible to match the truncated Taylor and exponential expansions at origin.

If we define the matrices $\mathbf{B}=\left(B_{i j}=b_{j}^{i}\right)_{i, j=1}^{N}$ $\mathrm{A}=\left(A_{i} \delta_{i j}\right)_{i, j=1}^{N}$ and $\mathbf{I}=\left(I_{i+j}\right)_{i, j=1}^{N}$ then we can rewrite equations (31) with $k=2,3, \ldots, 2 \mathrm{~N}$ as $\mathbf{I}=\mathbf{B A B}^{T}$ (where the superscript $T$ stands for transpose). Therefore, if the determinant of the square matrix in Eq. (36) vanishes then:

- one or more roots $b_{j}$ vanish;

- there are multiple roots $\left(b_{j}=b_{k}=\cdots\right)$;

- one or more coefficients $A_{j}$ vanish.

In any such case the approximation of order $N$ reduces to an approximation of lesser order.

It is not difficult to prove that

$$
S(t)^{2}=\frac{Z(t / 2)^{2}}{Z(t)}
$$

satisfies [9]

$$
\lim _{t \rightarrow \infty} S(t)^{2}=S_{\infty}^{2}=\left|\left\langle\phi \mid \psi_{0}\right\rangle\right|^{2}
$$

From

$$
\frac{d}{d t} \ln S(t)^{2}=E(t)-E(t / 2)
$$

one easily derives an approximation to the overlap in terms of the parameters of the exponential expansion:

$$
\ln S_{N}^{2}=\ln |\langle\phi \mid \phi\rangle|^{2}-\sum_{j=1}^{N} \frac{A_{j}}{b_{j}} .
$$

When $\langle\phi \mid \phi\rangle=1$ this expression agrees with the one derived by Cioslowski [9] except for the minus sign that is missing in his Eq. (21). Cioslowski did not use this expression directly but an equivalent one in terms of matrices built from the connected moments. Here we will use it in order to test the formulas derived above for the parameters of the exponential series. For generality we keep the term $\ln |\langle\phi \mid \phi\rangle|^{2}$ because in some cases our trial functions will not be normalized to unity.

\section{Illustrative examples}

In order to test the equations developed in the preceding section in what follows we apply them to some simple examples where we can carry out calculations of sufficiently large order.

Table 1. Exact overlaps between the trial functions (43) and the harmonic-oscillator eigenfunctions

\begin{tabular}{lll}
\hline$j$ & $\left|\left\langle\phi_{g} \mid \psi_{j}\right\rangle\right|^{2}$ & $\left|\left\langle\phi_{e} \mid \psi_{j}\right\rangle\right|^{2}$ \\
\hline \hline 0 & 1.969393167 & 0.006078373974 \\
2 & 0.01215674794 & 1.515878931 \\
4 & 0.0001125624810 & 0.05586468983 \\
6 & 0.000001158050216 & 0.001291015111 \\
\hline
\end{tabular}

We first consider the harmonic oscillator

$$
\hat{H}=-\frac{d^{2}}{d x^{2}}+x^{2}
$$

and the unnormalized trial functions

$$
\begin{aligned}
& \left\langle x \mid \phi_{g}\right\rangle=\exp \left(-\frac{2 x^{2}}{5}\right) \\
& \left\langle x \mid \phi_{e}\right\rangle=\left(x^{2}-\frac{1}{2}\right) \exp \left(-\frac{2 x^{2}}{5}\right)
\end{aligned}
$$

already chosen by Amore et al. [6] for their analysis of the convergence properties of the CMX. Tab. 1 shows the exact overlaps $\left|\left\langle\phi \mid \psi_{j}\right\rangle\right|^{2}, j=0,2,4,6$, for these two trial functions. We appreciate that $\left|\phi_{g}\right\rangle$ and $\left|\phi_{e}\right\rangle$ exhibit larger overlaps with the ground and second excited state, respectively.

Tab. 2 shows the parameters $W_{j}$ and $d_{j}$ for the trial function $\left|\phi_{g}\right\rangle$. The former converge (from above) towards the eigenvalues of the harmonic oscillator and the latter towards the exact overlaps shown in Tab. 1 in complete agreement with the general proof given in the preceding section.

Tab. 3 shows the parameters $A_{j}$ and $b_{j}, j=0,1,2,3$, for the second $C M X$ ansatz $U^{(N)}(t)$ proposed in the preceding section. Note that the exponential parameter $b_{0}$ tends to zero as $N$ increases suggesting that the CMX applies successfully to this problem. Tab. 4 shows the same parameters but with $b_{0}$ set equal to 0 as in the first approach $E^{(N)}(t)$. The results of both tables approach each other as $N$ increases.

Tab. 5 shows that the approximate overlap $S_{N}^{2}$ given by Eq. (41) for the unnormalized trial function $\left|\phi_{g}\right\rangle$ tends to the corresponding exact result in Tab.1. Cioslowski's approach [9] applies successfully to this example. 
Table 2. Parameters $W_{j}$ and $d_{j}$ for the harmonic oscillator and the trial function $\left|\phi_{g}\right\rangle$ (43)

\begin{tabular}{llllc}
\hline$N$ & \multicolumn{1}{c}{$W_{0} / d_{0}$} & \multicolumn{1}{c}{$W_{1} / d_{1}$} & \multicolumn{1}{c}{$W_{2} / d_{2}$} & $W_{3} / d_{3}$ \\
\hline \hline 2 & 1.00000699 & 5.006424635 & 9.368568397 & - \\
& 1.969404521 & 0.01216455133 & 0.00009457604481 & - \\
3 & 1.0000001 & 5.000187579 & 9.027741984 & 13.67205836 \\
& 1.96939336 & 0.01215747214 & 0.000112048591 & 0.000007675514617 \\
4 & 1.00000000 & 5.000004232 & 9.001287128 & 13.07302878 \\
& 1.969393169 & 0.01215677486 & 0.000112568657 & 0.000001130187743 \\
\hline
\end{tabular}

Table 3. Parameters $b_{j}$ and $A_{j}$ of the ansatz (34) for the harmonic oscillator and the trial function $\left|\phi_{g}\right\rangle$ (43)

\begin{tabular}{llllc}
\hline$N$ & \multicolumn{1}{c}{$b_{0} / A_{0}$} & \multicolumn{1}{c}{$b_{1} / A_{1}$} & \multicolumn{1}{c}{$b_{2} / A_{2}$} & $b_{3} / A_{3}$ \\
\hline \hline 2 & 0.002381248414 & 4.198837892 & - & - \\
& 1.00145413 & 0.02354586947 & - & - \\
3 & 0.00004431997162 & 4.010093259 & 8.439885 & - \\
& 1.000036479 & 0.02471528396 & 0.0002482361748 & - \\
4 & 0.000000731108 & 4.000338156 & 8.037169546 & 12.76249194 \\
& 1.000000709 & 0.02469399105 & 0.0003029268491 & 0.000002372560526 \\
\hline
\end{tabular}

The second column in Tab. 6 shows that the CMX approximants (36) converge rapidly towards the ground state as $N$ increases. This success is unsurprising in the light of the preceding analysis of the CMX parameters $b_{j}$. We obtain the same results from Eq. (33) and the parameters $A_{j}$ given in Tab. 4 derived from the general equations of the preceding section.

Tab. 7 shows the parameters $W_{j}$ and $d_{j}$ for the trial function $\left|\phi_{e}\right\rangle$. The former converge (from above) towards the eigenvalues of the harmonic oscillator and the latter towards the exact overlaps shown in Tab. 1. Since the overlap of the trial function with the second excited state is larger than the overlap with the ground state we expect an anomalous behaviour of both ansätze $E^{(N)}$ and $U^{(N)}$ as discussed by Amore et al. [6]. This is in fact the case and some of the parameters $b_{j}$ for this trial function are negative or complex. However, the second ansatz $U^{(N)}$ discussed in the preceding section exhibits a small exponential parameter $b_{0}$ that appears to tend to zero as $N$ increases. At the same time, the corresponding coefficient $A_{0}$ tends to the energy of the second excited state as $N$ increases as shown in Tab. 8. This behaviour is consistent with the convergence of the CMX sequence (36) to the second excited state shown in the third column of Tab. 6 and discussed earlier by Amore et al [6]. Note that the CMX does not provide bounds to the energies as the Rayleigh-Ritz method already does.
As a nontrivial example we choose the simple anharmonic oscillator

$$
\hat{H}=-\frac{d^{2}}{d x^{2}}+x^{4}
$$

and the unnormalized trial functions

$$
\begin{aligned}
& \left\langle x \mid \phi_{g}\right\rangle=\exp \left(-\frac{3 x^{2}}{2}\right) \\
& \left\langle x \mid \phi_{e}\right\rangle=\left(x^{2}-\frac{1}{4}\right) \exp \left(-\frac{3 x^{2}}{2}\right)
\end{aligned}
$$

also considered by Amore et al. [6]. This oscillator is strongly anharmonic and enables us to calculate as many terms as desired for all the approximants discussed above.

Tab. 9 shows the parameters $W_{j}$ and $d_{j}, j=0,1,2$ for the trial function $\left|\phi_{g}\right\rangle$. The former converge (from above) towards the well known eigenvalues as $N$ increases and the latter provide the overlaps. Since the overlap with the ground state dominates the CMX will converge towards this state [6]. The second column of Tab. 10 shows the great rate of convergence of the CMX sequence (36) towards the ground state of the anharmonic oscillator, already calculated by Amore et al. [6]. Once again we appreciate that the $\mathrm{CMX}$ does not provide bounds.

Tab. 11 shows the parameters for the ansatz $U^{(N)}$. The parameter $b_{0}$ tends to zero and $A_{0}$ towards the energy of the ground state of the anharmonic oscillator as $N$ increases. However, spurious roots $b_{j}$ and values of the corresponding parameters $A_{j}$ appear when $N \geq 3$. We have just 
Table 4. Parameters $b_{j}$ and $A_{j}$ of the ansatz (30) for the harmonic oscillator and the trial function $\left|\phi_{g}\right\rangle$ (43)

\begin{tabular}{lllc}
\hline$N$ & \multicolumn{1}{c}{$b_{1} / A_{1}$} & \multicolumn{1}{c}{$b_{2} / A_{2}$} & $b_{3} / A_{3}$ \\
\hline \hline 2 & 4.003491206 & 8.296508793 & - \\
& 0.02472188733 & 0.0002743493113 & - \\
3 & 4.000086984 & 8.019485444 & 12.58042783 \\
& 0.02469258182 & 0.0003046174966 & 0.000002754219691 \\
4 & 4.000001796 & 8.000821311 & 12.05918785 \\
& 0.02469138991 & 0.0003048780599 & 0.000003706290872 \\
\hline
\end{tabular}

Table 5. Overlap for the ground state of the harmonic oscillator from Eq. (41)

\begin{tabular}{ll}
\hline$N$ & $S_{N}^{2}$ \\
\hline \hline 2 & 1.969399291 \\
3 & 1.969393256 \\
4 & 1.969393168 \\
\hline
\end{tabular}

Table 6. Convergence of the $\mathrm{CMX}$ for the harmonic oscillator and the two trial functions $\left|\phi_{g}\right\rangle$ and $\left|\phi_{e}\right\rangle$ (43)

\begin{tabular}{lll}
\hline$N$ & $A_{0, N}(g)$ & $A_{0, N}(e)$ \\
\hline \hline 1 & 1.000304878 & 4.931822888 \\
2 & 1.000003763 & 5.014793896 \\
3 & 1 & 5.002413906 \\
4 & 1 & 5.001402117 \\
5 & 1 & 4.999955757 \\
6 & 1 & 5.002955554 \\
7 & 1 & 5.000013363 \\
8 & 1 & 5.000011300 \\
9 & 1 & 5.000001215 \\
10 & 1 & 4.999999154 \\
\hline
\end{tabular}

chosen those that follow the reasonable sequences determined by the results for smaller values of $N$.

Tab. 12 shows the parameters for the ansatz $E^{(N)}$. Note that the agreement between the parameters of the two ansätze for the anharmonic oscillator is not as good as in the case of the harmonic one. In this case we also obtain apparently spurious roots $b_{j}$ and coefficients $A_{j}$ for $N>4$. For example, when $N=6 b_{2}$ and $A_{2}$ are the complex conjugates of $b_{3}$ and $A_{3}$, respectively. Consequently, the complex parts of $A_{2}$ and $A_{3}$ cancel each other in Eq. (33) that yields a reasonable approach to the ground-state energy $A_{0}=1.0603680$. We conclude that the parameters $A_{j}$ and $b_{j}$ should not necessarily be real and positive for the CMX approximants (36) to converge neatly towards the ground-state energy.
Tab. 13 shows that the overlap between the ground state of the anharmonic oscillator and $\left|\phi_{g}\right\rangle$ calculated by means of Eq. (41) agrees with the result of Tab. $9\left(d_{0}\right)$. Once again we realize that the complex parts of the parameters $A_{j}$ and $b_{j}$ cancel out to produce a reasonable real approximation to the expected result. The occurrence of complex parameters in the exponential ansatz is not revealed by the CMX approximants (like Eq. (36)) based on the connected moments.

As a two-dimensional example we choose the PullenEdmonds Hamiltonian

$$
\hat{H}=-\frac{1}{2} \nabla^{2}+\frac{1}{2}\left(x^{2}+y^{2}\right)+\alpha x^{2} y^{2}
$$

that has been already treated by the CMX [10], the generalized moments expansion (GMX) and the canonical sequence method (CSM) [11]. A reasonable trial function for the ground state is given by the unnormalized Gaussian function

$$
\langle x, y \mid \phi\rangle=e^{-a\left(x^{2}+y^{2}\right)}
$$

where $a>0$. Cioslowski [10] obtained satisfactory results for $0.1 \leq \alpha \leq 0.5$ with the CMX and $a=1 / 2$.

Tab. 14 shows the exponents $b_{j}$ for $\alpha=0.5$ (the greatest value considered by Cioslowski [10] and Fessatidis et $a l$. [11]), and two values of $a(a=0.5$ and $a=1)$. Since all the exponents are positive we expect that the CMX will give satisfactory results for both values of $a$. However, note that the sequence of values of $b_{0}$ converges towards 0 more rapidly when $a=1$ so that we also expect the rate of convergence of the CMX to be greater for this case.

Tab. 15 shows the CMX results for the ground-state energy of the Pullen-Edmonds Hamiltonian for both values of $a$. The rate of convergence is greater for $a=1$ as suggested by the analysis of the convergence of the exponent $b_{0}$. The entries in the second column are similar to Cioslowski's results [10] (note that Cioslowski' index $n$ is equal to present $N+1$ ) and those in the third column correspond to $a=1$. The last two rows show the results provided by the GMX $(\mathrm{GMX}(1,3)$, fifth order) and 
Table 7. Parameters $W_{j}$ and $d_{j}$ for the harmonic oscillator and the trial function $\left|\phi_{e}\right\rangle(43)$

\begin{tabular}{llrlc}
\hline$N$ & \multicolumn{1}{c}{$W_{0} / d_{0}$} & $W_{1} / d_{1}$ & \multicolumn{1}{c}{$W_{2} / d_{2}$} & \multicolumn{1}{c}{$W_{3} / d_{3}$} \\
\hline \hline 2 & 2.911817131 & 5.079618783 & 9.870638470 & - \\
& 0.04317577925 & 1.498883666 & 0.03707877419 & - \\
3 & 1.060922282 & 5.002758941 & 9.097824481 & 14.14651589 \\
& 0.006448836455 & 1.517139667 & 0.05488390863 & 0.0006658074829 \\
4 & 1.001339803 & 5.000101462 & 9.007425114 & 13.18333763 \\
& 0.006087180960 & 1.515949720 & 0.05588279435 & 0.001209845824 \\
5 & 1.000026913 & 5.000003052 & 9.000381181 & 13.01903413 \\
& 0.006078565317 & 1.515881592 & 0.05586900205 & 0.001287657490 \\
\hline
\end{tabular}

Table 8. Parameters $b_{0}$ and $A_{0}$ for the harmonic oscillator with the trial function $\left|\phi_{e}\right\rangle$ (43)

\begin{tabular}{ccc}
\hline$N$ & $b_{0}$ & $A_{0}$ \\
\hline \hline 2 & 0.01634078866 & 5.013227071 \\
3 & -0.002960622766 & 4.999388680 \\
4 & 0.006737033331 & 4.997247173 \\
5 & 0.0003889752190 & 5.000207908 \\
\hline
\end{tabular}

Table 9. Parameters $W_{j}$ and $d_{j}$ for the anharmonic oscillator (44) and the trial function $\left|\phi_{g}\right\rangle$ (45)

\begin{tabular}{llll}
\hline$N$ & \multicolumn{1}{c}{$W_{0} / d_{0}$} & \multicolumn{1}{c}{$W_{1} / d_{1}$} & \multicolumn{1}{c}{$W_{2} / d_{2}$} \\
\hline \hline 2 & 1.069780255 & 7.871169487 & 20.45762861 \\
& 0.9487351539 & 0.07314504796 & 0.001446505981 \\
3 & 1.061229046 & 7.516944429 & 17.25938517 \\
& 0.9451196068 & 0.07523345172 & 0.002946453665 \\
4 & 1.060427446 & 7.462353629 & 16.44650531 \\
& 0.9447200769 & 0.07517101138 & 0.003352072665 \\
5 & 1.06036628 & 7.456258219 & 16.28617073 \\
& 0.944686457 & 0.07513217688 & 0.003396340115 \\
\hline
\end{tabular}

Table 10. Convergence of the $\mathrm{CMX}$ towards the ground-state $(g)$ and second-excited state $(e)$ energies of the anharmonic oscillator (44)

\begin{tabular}{rll}
\hline$M$ & $A_{0}(g)$ & $A_{0}(e)$ \\
\hline \hline 5 & 1.060692159 & 7.439371257 \\
10 & 1.060363186 & 7.456069907 \\
15 & 1.060362073 & 7.450017954 \\
20 & 1.060362093 & 7.451366303 \\
25 & 1.060362090 & 7.455118704 \\
30 & 1.060362090 & 7.454183973 \\
35 & " & 7.451642486 \\
40 & " & 7.454364274 \\
50 & " & 7.454214745 \\
60 & " & 7.453864737 \\
70 & " & 7.455066766 \\
80 & " & 7.455185890 \\
90 & " & 7.453941990 \\
100 & " & 7.453833053 \\
\hline exact & 1.060362090 & 7.455697938 \\
\hline
\end{tabular}


Table 11. Parameters $b_{j}$ and $A_{j}$ of the ansatz (34) for the anharmonic oscillator (44) with the trial function $\left|\phi_{g}\right\rangle$ (45)

\begin{tabular}{cllc}
\hline$N$ & \multicolumn{1}{c}{$b_{0} / A_{0}$} & \multicolumn{1}{c}{$b_{1} / A_{1}$} & \multicolumn{1}{c}{$b_{2} / A_{2}$} \\
\hline \hline 2 & 0.08325285817 & 6.885234502 & 20.86679836 \\
& 1.099547538 & 0.4729461665 & 0.01083962814 \\
3 & 0.001993821505 & 6.359516968 & 17.31475086 \\
& 1.06101123 & 0.4997973255 & 0.02232719076 \\
4 & -0.003775464406 & 6.306016728 & 16.74692001 \\
& 1.057884464 & 0.5006911384 & 0.02434537896 \\
\hline
\end{tabular}

Table 12. Parameters $b_{j}$ and $A_{j}$ of the ansatz (30) for the anharmonic oscillator (44) with the trial function $\left|\phi_{g}\right\rangle$ (45)

\begin{tabular}{llcl}
\hline$N$ & \multicolumn{1}{c}{$b_{1} / A_{1}$} & \multicolumn{1}{c}{$b_{2} / A_{2}$} & \multicolumn{1}{c}{$b_{3} / A_{3}$} \\
\hline \hline 2 & 6.470844472 & 19.19699588 & - \\
& 0.503507803 & 0.01645848089 & - \\
3 & 6.343642152 & 17.18884282 & 34.42060985 \\
& 0.5002800387 & 0.02285212523 & 0.0002229752367 \\
4 & 6.342827268 & 17.16971044 & 34.25122888 \\
& 0.5002367582 & 0.02290363548 & 0.0002309382854 \\
5 & 5.692034185 & 5.980920104 & 16.54089332 \\
& -0.5783779771 & 1.075044172 & 0.02550181868 \\
\hline
\end{tabular}

Table 13. Overlap for the ground state of the anharmonic oscillator from Eq. (41)

\begin{tabular}{ll}
\hline$N$ & $S_{N}^{2}$ \\
\hline \hline 2 & 0.9459076757 \\
3 & 0.9444614836 \\
4 & 0.9444538767 \\
5 & 0.9449417075 \\
6 & 0.9446880500 \\
\hline
\end{tabular}

Table 14. Exponents $b_{j}$ for the Pullen-Edmonds Hamiltonian with $\alpha=0.5$ for two values of $a$

\begin{tabular}{lllccc}
\hline \multicolumn{5}{c}{$\begin{array}{c}a=1 / 2 \\
a=1\end{array}$} \\
\hline$N$ & \multicolumn{1}{c}{$b_{0}$} & \multicolumn{1}{c}{$b_{2}$} & $b_{3}$ & $b_{4}$ \\
\hline \hline 2 & 0.02906068543 & 8.255003979 & & & \\
& 0.05137853443 & 3.179278970 & & & \\
3 & 0.01302652881 & 6.306411183 & 21.79335651 & & \\
& 0.005884721637 & 2.588321084 & 6.835685938 & & \\
4 & 0.007719495186 & 5.474848514 & 15.83509229 & 42.12030301 & \\
& 0.0008448771176 & 2.469668165 & 5.742988280 & 10.94038086 & \\
5 & 0.005264561288 & 4.956739629 & 12.87136665 & 30.93888458 & 69.90721593 \\
& 0.0002110290626 & 2.441442453 & 5.283795070 & 8.720985401 & 14.71987930 \\
6 & 0.003841243108 & 4.558996554 & 10.98579271 & 25.27139705 & 52.38567030 \\
& 0.00005030482040 & 2.427133379 & 4.784038202 & 7.033461737 & 11.56668194 \\
\hline
\end{tabular}


its cousin the CSM $(\operatorname{CSM}(13))$ that Fessatidis et al. [11] considered to be excellent. However, Tab. 15 clearly reveals that at least for this model neither the GMX nor the CSM appear to improve the results of the much simpler and more straightforward CMX.

Table 15. Ground-state energy for the Pullen-Edmonds with $\alpha=0.5$

\begin{tabular}{rll}
\hline$N$ & \multicolumn{1}{c}{$a=1 / 2$} & \multicolumn{1}{c}{$a=1$} \\
\hline \hline 2 & 1.101104251 & 1.09896838 \\
3 & 1.09978076 & 1.09810749 \\
4 & 1.099074971 & 1.097991618 \\
5 & 1.098653642 & 1.097963238 \\
6 & 1.098396191 & 1.097958951 \\
7 & 1.098238541 & 1.097958772 \\
8 & 1.098141493 & 1.097958746 \\
9 & 1.098080974 & 1.097959376 \\
10 & 1.098042519 & 1.097959059 \\
11 & 1.098017546 & 1.097959037 \\
12 & 1.098000958 & 1.097959034 \\
13 & 1.097989693 & 1.097959034 \\
14 & 1.097981882 & 1.097959033 \\
15 & 1.097976358 & 1.097959016 \\
16 & 1.097972383 & 1.097959047 \\
17 & 1.097969474 & 1.097959043 \\
18 & 1.097967314 & 1.097959042 \\
19 & 1.097965688 & 1.097959042 \\
\hline$E_{0}^{C S M}(13)$ & 1.11022 & \\
\hline
\end{tabular}

\section{Conclusions}

In this paper we propose a systematic procedure for solving the nonlinear equations that appear when matching a Taylor series about $t=0$ and an asymptotic exponential expansion valid for large $t$. We applied it to the analysis of the extrapolation of the $t$-expansions for the generating functions of the moments and connected moments. Obviously, $Z(t)$ is more suitable for matching both expansions at origin because this function does not exhibit singular points. Unfortunately, results coming from it are not size consistent. On the other hand $E(t)$ exhibits singularities at the zeroes of $Z(t)$ in the complex $t$-plane that may hinder the extrapolation (see also Amore et al. [6] for other examples). The full solution of the nonlinear equations enables us to test whether the main assumptions of the CMX are valid for the reference state chosen for the study of a given quantum-mechanical problem. We have analyzed two cases for the harmonic oscillator and two more for an anharmonic one and have shown that the CMX equations yield better results for the former which is not surprising. We have also seen that the rate of convergence of the CMX approximants (36) may be large even when the parameters in the ansatz $E^{(N)}(t)$ are complex. This most interesting feature of the CMX was not revealed by earlier applications of the approach because they were based on algorithms that bypass the explicit calculation of the parameters of the ansatz $E^{(N)}(t)$.

Knowles' equation (36) for the energy and Cioslowski's equation (41) for the overlap (in terms of the connected moments [9]) are remarkable ways of bypassing the explicit calculation of the apparently unnecessary variables in the nonlinear equation (5). However, we have shown that it is not difficult to calculate all those variables explicitly and obtain additional information on the behaviour of the approach.

We want to point out that the main results of sections 2 and 3 are quite general and apply to any quantummechanical problem. In Section 4 we have chosen extremely simple models with the only purpose of facilitating the calculation of the necessary moments (which appears to be the main difficulty in the application of all the moments methods). Even the treatment of a two-dimensional oscillator offers no difficulty as illustrated in Section 4.

The main results of Section 2 are not restricted to the analysis of the generating functions for the moments and connected moments. In future works we will explore their utility in other problems of physical interest. Just to mention one example, note that $Z(i t)=$ $\langle\psi(0)|\exp (-i t \hat{H})| \psi(0)\rangle$ is the projection of the state at time $t|\psi(t)\rangle=\exp (-i t \hat{H})|\psi(0)\rangle$ onto the initial state $|\psi(0)\rangle$ (correlation function). We easily obtain $Z(i t)$ for the harmonic and anharmonic oscillators from the results of tables 2, 7 and 9 .

\section{Acknowledgements}

P.A. acknowledges support of Conacyt through the SNI fellowship and also of PIFI. F.M.F acknowledges support of PIFI and of UNLP through the "subsidio para viajes $y / 0$ estadías"

\section{References}

[1] D. Horn, M. Weinstein, Phys. Rev. D 30, 1256 (1984)

[2] D. Horn, M. Karliner, M. Weinstein, Phys. Rev. D 31, 2589 (1985)

[3] J. Cioslowski, Phys. Rev. Lett. 58, 83 (1987)

[4] C. Stubbins, Phys. Rev. D 38, 1942 (1988)

[5] P. Knowles, Chem. Phys. Lett. 134, 512 (1987)

[6] P. Amore, F. M. Fernández, M. Rodriguez, J. Phys. A 44, 505302 (2011) 
[7] F. M. Fernández, Int. J. Quantum Chem. 109, 717 (2009)

[8] I. Bartashevich, Int. J. Quantum Chem. 108, 272 (2008)

[9] J. Cioslowski, Phys. Rev. A 36, 3441 (1987)
[10] J. Cioslowski, Chem. Phys. Lett. 136, 515 (1987)

[11] V. Fessatidis, J. D. Mancini, S. P. Bowen, M. Campuzano, J. Math. Chem. 44, 20 (2008) 